Editorial

\title{
Rabb-Venable Excellence in Ophthalmology Research Program: Contributions to Ophthalmology Workforce Diversity
}

\author{
Mildred M.G. Olivier, MD ${ }^{4}$ Eydie G. Miller-Ellis, MD 5 \\ ${ }^{1}$ Department of Ophthalmology, The University of North Carolina at \\ Chapel Hill, Chapel Hill, North Carolina \\ 2 Department of Ophthalmology and Visual Sciences, Vanderbilt \\ University, Nashville, Tennessee \\ ${ }^{3}$ And So It Begins, LLC \\ ${ }^{4}$ Department of Surgery, Chicago Medical School, Rosalind Franklin \\ University of Medicine and Science, North Chicago, Illinois \\ ${ }^{5}$ Department of Ophthalmology, University of Pennsylvania, \\ Philadelphia, Pennsylvania
}

O’Rese J. Knight, MD ${ }^{10}$ Dolly Ann Padovani-Claudio, MD, PhD² Clarisse C. Croteau-Chonka, PhD ${ }^{3}$

J Acad Ophthalmol 2021;13:e298-e303.

The 2016 Health Resources and Services Administration, Health Workforce Analysis estimated that ophthalmology is the surgical specialty with the greatest physician shortage. Based on their estimates, the ophthalmology workforce needs to expand by $33 \%$ to 22,690 full time equivalents to meet its 2025 patient care demand. ${ }^{1}$ Despite the need to expand the ophthalmology workforce, the number of underrepresented in medicine (URiM) ophthalmology trainees decreased from 8.7 to $7.7 \%$ between 2005 and 2015 . $^{2}$ Patients from underrepresented groups, including Black/African Americans, Latinx, American Indians, Alaskan Natives, Native Hawaiians, and Pacific Islanders, comprised $30.7 \%$ of the U.S. population in the same period. - Table 1 summarizes the racial/ethnic composition of the U.S. population, current ophthalmology workforce, and ophthalmology trainee pathway. The disproportionate ratio of URiM ophthalmologists compared with patients likely accounts for a significant portion of the racial/ethnic disparities in care delivery and eye health outcomes. ${ }^{3-8}$ Ample evidence demonstrates that health care utilization and care outcomes improve with patient-physician racial concordance and that URiM physicians are more likely to work in medically underserved communities. ${ }^{9,10}$ However, despite an increase in the total number of ophthalmology residency training positions offered through the SF Match to meet the patient care demand, ${ }^{11}$ the number of URiM physicians matching in ophthalmology residency programs between 2016 and 2020 remained flat at $8.4 \%$ per year. $^{12}$ As the number of ophthalmology residency training positions increases, specific effort should be made to recruit talented URiM medical students into ophthalmology. Racial concordance is associated with increased cultural competency (providing culturally and linguistically appropriate services) which is expected to reduce racial and ethnic health disparities and improve the quality of health care. ${ }^{7}$ The main goal of Rabb-Venable (RV) is to implement interventions to recruit URiM trainees into ophthalmology to reflect the cultural diversity of the community receiving eye care services. This is expected to translate into increased cultural competency and better patient outcomes.

\section{Barriers to Entry}

URiM applicants to medical school are more likely to be first generation in medicine and/or college as suggested by their greater likelihood to have parental combined gross incomes less than $\$ 50 \mathrm{~K}$, premedical education debt, and greater amounts of indebtedness. ${ }^{13}$ During medical school, students average just 12.5 hours of preclinical ophthalmology coursework. ${ }^{14}$ The top three reasons medical students do not choose ophthalmology as a career include: "insufficient interest," "too specialized," and "not enough exposure." Insufficient same race or same gender role models or mentorship is a barrier to exposure, and is of significantly greater concern for URiM than for non-URiM students. ${ }^{15}$ Howard University, University of Illinois, University of Puerto Rico,
Address for correspondence O'Rese J. Knight, MD, Department 10.1055/s-0041-1736215. of Ophthalmology, The University ISSN 2475-4757. of North Carolina at Chapel Hill, 5154 Bioinformatics Bldg, CB: \# 7040, Chapel Hill, NC 27599-7040 (e-mail: orese_knight@med.unc.edu).
DOI https://doi.org/ 40

\footnotetext{
(C) 2021. The Author(s).

This is an open access article published by Thieme under the terms of the Creative Commons Attribution-NonDerivative-NonCommercial-License, permitting copying and reproduction so long as the original work is given appropriate credit. Contents may not be used for commercial purposes, or adapted, remixed, transformed or built upon. (https://creativecommons.org/ licenses/by-nc-nd/4.0/) Thieme Medical Publishers, Inc., 333 Seventh Avenue, 18th Floor, New York, NY 10001, USA
} 
Table 1 Race/ethnicity of the U.S. population, current ophthalmology workforce, and the trainee pathway: the 2019 American Community Survey estimates that $31.6 \%$ of the U.S. population are from underrepresented in medicine (URiM) groups, while URiM physicians comprise $6.7 \%$ of the current ophthalmology workforce and were awarded $9.7 \%$ of the 2019 ophthalmology residency positions

\begin{tabular}{|c|c|c|c|c|c|c|c|c|}
\hline \multirow[t]{2}{*}{ Group } & \multicolumn{8}{|l|}{ Race/Ethnicity } \\
\hline & $\begin{array}{l}\text { American } \\
\text { Indian } \\
\text { and Alaska } \\
\text { Native }\end{array}$ & Asian & $\begin{array}{l}\text { Black or } \\
\text { African/ } \\
\text { American }\end{array}$ & Latin $x$ & $\begin{array}{l}\text { Native } \\
\text { Hawaiian } \\
\text { and Other } \\
\text { Pacific } \\
\text { Islander }\end{array}$ & White & URiM & Total \\
\hline \multirow[t]{2}{*}{2019 US Census estimate ${ }^{a}$} & $2,236,348$ & $18,427,914$ & $40,596,040$ & $60,481,746$ & 565,473 & $196,789,401$ & $103,879,607$ & $328,239,523$ \\
\hline & $0.7 \%$ & $5.6 \%$ & $12.4 \%$ & $18.4 \%$ & $0.2 \%$ & $60.0 \%$ & $31.6 \%$ & \\
\hline \multirow[t]{2}{*}{ Ophthalmology Workforce $(2018)^{\mathrm{b}}$} & 24 & 3396 & 512 & 726 & 13 & 11587 & 1,275 & 19,085 \\
\hline & $0.1 \%$ & $17.8 \%$ & $2.7 \%$ & $3.8 \%$ & $0.1 \%$ & $60.7 \%$ & $6.7 \%$ & \\
\hline \multirow[t]{2}{*}{ Undergraduate Enrollment (2015) ${ }^{c}$} & 194,486 & $1,285,969$ & $3,363,643$ & $3,918,192$ & 69,079 & $12,084,691$ & $7,545,400$ & $23,620,218$ \\
\hline & $0.8 \%$ & $5.4 \%$ & $14.2 \%$ & $16.6 \%$ & $0.3 \%$ & $51.2 \%$ & $31.9 \%$ & \\
\hline \multirow[t]{2}{*}{ Medical School Applicants (2015) ${ }^{\mathrm{d}}$} & 115 & 10,122 & 4,087 & 3,219 & 50 & 25,101 & 7,471 & 52,550 \\
\hline & $0.2 \%$ & $19.3 \%$ & $7.8 \%$ & $6.1 \%$ & $0.1 \%$ & $47.8 \%$ & $14.2 \%$ & \\
\hline \multirow[t]{2}{*}{ Medical School Matriculants (2015) ${ }^{\mathrm{d}}$} & 20 & 3701 & 1061 & 854 & 5 & 10992 & 1,940 & 18,705 \\
\hline & $0.1 \%$ & $19.8 \%$ & $5.7 \%$ & $4.6 \%$ & $0.03 \%$ & $58.8 \%$ & $10.4 \%$ & \\
\hline \multirow[t]{2}{*}{ Medical School Graduates (2019) ${ }^{\mathrm{e}}$} & 38 & 4,299 & 1,238 & 1,063 & 9 & 10,879 & 2,348 & 19,937 \\
\hline & $0.2 \%$ & $21.6 \%$ & $6.2 \%$ & $5.3 \%$ & $0.0 \%$ & $54.6 \%$ & $11.8 \%$ & \\
\hline \multirow[t]{2}{*}{ SF Match Participants (2019) ${ }^{f}$} & - & 181 & 21 & 48 & - & 305 & 69 & 588 \\
\hline & - & $30.8 \%$ & $3.6 \%$ & $8.2 \%$ & - & $51.9 \%$ & $11.7 \%$ & \\
\hline \multirow[t]{2}{*}{ Ophthalmology Positions (2019) ${ }^{\mathrm{f}}$} & - & 124 & 13 & 34 & - & 239 & 47 & 437 \\
\hline & - & $28.4 \%$ & $3.0 \%$ & $7.8 \%$ & - & $54.7 \%$ & $10.8 \%$ & \\
\hline
\end{tabular}

a2019 American Community Survey 1-Year Estimates Data

${ }^{\mathrm{b}}$ AMA Physician Masterfile, 2018

'National Center for Education Statistics 12-month unduplicated headcount at Title IV institutions

${ }^{\mathrm{d}}$ AAMC Facts \& Figures 2016

${ }^{\mathrm{e} A A M C}$ Diversity in Medicine: Facts and Figures 2019

${ }_{\mathrm{f}}^{\mathrm{A}} \mathrm{AUPO} / \mathrm{SF}$ Match Gender \& Ethnicity Data: Ophthalmology Residency 2019

and University of Texas Medical Branch are the four medical institutions that have large URiM student populations and academic ophthalmology departments. ${ }^{13}$ - Table 2 lists the top five medical schools that have graduated the most URiM physicians between 2010 and 2019. Note that the medical schools that train the most URiM physicians may not have departments of ophthalmology. To address some of these barriers, the American Academy of Ophthalmology (AAO) developed the Minority Ophthalmology Mentoring (MOM) program which pairs ophthalmology mentors with interested preclinical medical students and college students across the nation. Aside from lack of exposure and access to role models, URiM student's performance on United States Medical Licensing Examination (USMLE) Step 1 likely presents a more significant hurdle.

The average USMLE Step 1 score for Black/African American and Latinx students between 2010 and 2015 was 217 and 221 , respectively, ${ }^{16}$ during that same period the average score for ophthalmology applicants who did not match rose from 214 to $228 .^{11}$ Several residency programs across specialties have demonstrated the negative effect that using USMLE examination scores has on the diversity of their

Table 2 U.S. Medical Schools with the most URiM graduates 2010 to 2019

\begin{tabular}{|l|l|l|}
\hline Black/African American & Latinx & American Indian/Alaskan Native \\
\hline Meharry Medical College & University of Puerto Rico & University of Oklahoma \\
\hline Howard University & Ponce Health Sciences University & University of Minnesota \\
\hline Morehouse University & Universidad Central del Caribe & University of New Mexico \\
\hline University of Illinois & University of Illinois & University of North Dakota \\
\hline University of Texas Medical Branch & San Juan Bautista & University of Washington \\
\hline
\end{tabular}

Abbreviation: URiM, underrepresented in medicine.

Note: These are the top 5 medical schools that graduate Black/African American, Latinx, and American Indian/Alaskan Native physicians, respectively. The institutions that are bolded have departments of ophthalmology. 


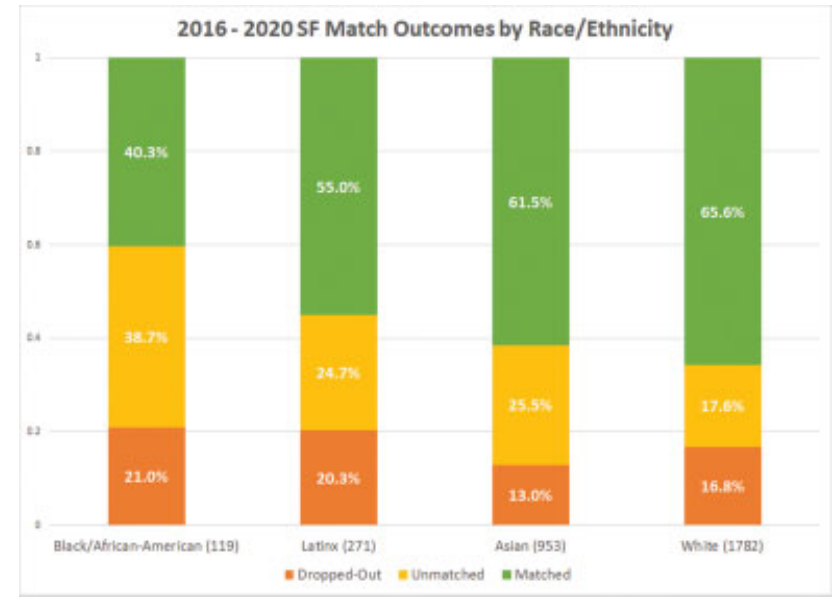

Fig. 12016 to 2020 SF Match outcomes by race/ethnicity: The total number of applicants who registered for SF Match are Black/African American (119), Latinx (271), Asian (953), and white (1,782). Five American Indian/Alaskan Native physicians registered, three submitted rank lists, and two matched. One Native Hawaiian/Pacific Islander registered in 2019 but did not submit a rank list or match. This analysis does not include applicants who declined to state their race/ethnicity or who identified as two or more races.

applicant pools. ${ }^{17-22}$ During the 2016 to 2020 match cycles, the percentage of applicants who were rejected, defined as the number of registrants who did not receive an interview divided by the number of registrants, was 21.0 (25/119) and 20.3\% (55/271) for Black/African American and Latinx applicants, respectively, versus 13.0 (124/953) and 16.8\% (299/1782) for Asian and white applicants, respectively. The average match rates between 2016 and 2020, defined as the number of participants awarded a residency position divided by the number of applicants that completed interviews for Black/African American, Latinx, Asian, and white applicants were 51.1 (48/94), 69.0 (149/216), 70.7 (586/829), and $78.8 \%(1169 / 1483)$, respectively ( - Fig. 1 ). ${ }^{12}$ If the rejection and match rates for white applicants was the same for all groups, 82 Black/African American and 187 Latinx applicants would have matched. The net effect of disparate rejection and match rates related to race/ethnicity result is the loss of $70.8 \%$ (82 possible/48 actual) of Black/African American and 25.5\% (187 possible/149 actual) of Latinx physicians who could have joined the ophthalmology workforce. The lack of exposure to ophthalmology and the use of USMLE Step 1 scores as the primary criterion for screening applications may be the two most significant factors accounting for the disparity between increased number of URiM students and stagnant number of URiM ophthalmology residents despite the increased number of ophthalmology training positions. To address this need, the AAO MOM program also provides access to USMLE precourse and study materials to participants. MOM targets students interested in ophthalmology at the pre-med and first year of medical school level as a recruitment tool. The RV program follows through that mission to the remainder of URiM medical school training with the goal to increase the proportion of competitive and successful URiM ophthalmology residency applicants.

\section{Rabb-Venable Program Outcomes}

The Rabb-Venable Excellence in Ophthalmology Research Program was formalized in 2000 by the Ophthalmology Section of the National Medical Association (NMA). The program is named for Maurice F. Rabb, Jr., MD, and Howard P. Venable, MD, in honor of their prolific support of URiM's pursuing careers in academic ophthalmology. RV has been funded by a combination of education grants from industry and a R13 Travel Grant from the National Eye Institute (NEI). URiM medical students are recruited at the Student National Medical Association Annual Medical Education Conferences, Latino Medical Student Association National Conferences, and Society for Advancement of Chicanos/Latinx and Native Americans in Science Conferences, and Association of Native American Medical Student events. In addition, URiM ophthalmology trainees (residents and fellows) are recruited directly by their department or through the growing network of RV alumni, or through our leadership connections with the MOM program.

Traditionally, trainees have submitted an abstract of their original research to be judged by members of the RabbVenable Scientific Committee. The best abstracts are selected for either oral or poster presentations at the annual NMA Ophthalmology Section scientific meeting where presenters field questions from the entire NMA Ophthalmology Section membership. The medical students who are not selected to present are invited to participate as observers and encouraged to identify potential research mentors during the meeting. Observers often return to present their work in subsequent years, after completing research with their mentors. The winners of the RV research program are sponsored to present their work at the annual Association for Research in Vision and Ophthalmology or other

Rabb-Venable Program Outcomes

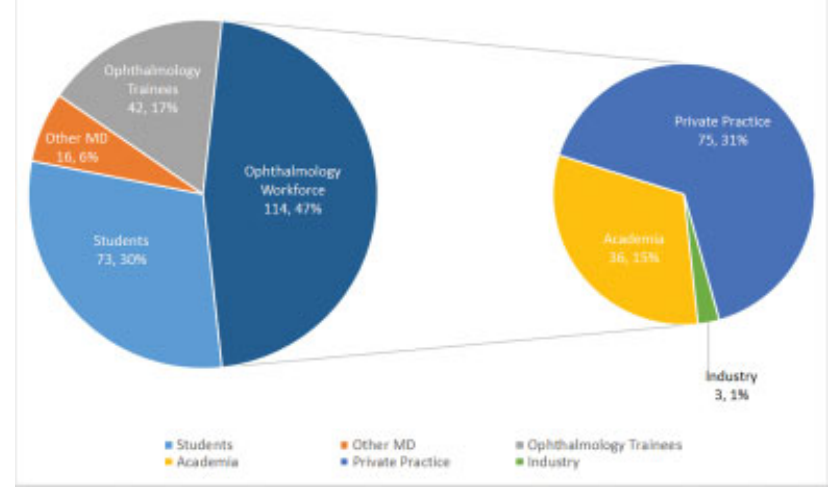

Fig. 2 Rabb-Venable Program Outcomes: Since 2000, the RabbVenable Program has had 245 participants, of which only 16 (6\%) are working in medical subspecialties other than ophthalmology. Seventy-three (30\%) are in preresidency training and $42(17 \%)$ are completing their ophthalmology training. One hundred and thirteen (46\%) are already practicing ophthalmology, of which $31.5 \%$ hold academic positions. Eight additional Rabb-Venable participants could not be verified. 
ophthalmology meetings. Participants are encouraged to return in subsequent years to present new research.

In addition to showcasing trainee research, RV provides formal professional development, mentorship, and community service opportunities. The professional development components include speaker and interview training with a professional communication coach, NEI grant funding and research opportunities workshops with an NEI program officer, and keynote presentations with RV alumni in academic or private practice. Mentorship opportunities include a welcome dinner with RV alumni who are junior faculty involved in the residency selection process to discuss preparation for the SF Match process and pairing with NMA members for further research and career opportunities. Community service opportunities often include participation in vision screenings and career fairs for K-12 students in the host cities of the NMA Convention.

Since 2000, RV has hosted 245 unique participants: 73 are current medical students, 42 are completing ophthalmology residencies and fellowships, and 114 are practicing physicians. The ophthalmology match rate for eligible RV participants between 2008 and 2020 is 75\%. Only 16 (6\%) RV participants have chosen a specialty other than ophthalmology. Additionally, 31.5\% of RV participants who have completed their ophthalmology training hold academic positions (-Fig. 2). Several have NEI funded research and many serve in leadership roles within national ophthalmology organizations and their respective departments, as program directors, medical student educators, diversity officers, and division chiefs.

\section{Response to COVID-19}

The RV program celebrated its 20th anniversary in 2020. Given the high degree of penetration RV has achieved in recruiting URiM students to ophthalmology, it was necessary to adapt the program proceedings to the coronavirus disease 2019 (COVID-19)-imposed travel restrictions. Not only were senior medical students at risk of not participating in RV but they also lost their opportunities to complete additional clinical and research training activities, explore residency programs of interest, and seek additional letters of recommendation. The Coalition for Physician Accountability discouraged away rotations for the duration of the 2020 to 2021 academic year ${ }^{23}$ and COVID-19-related financial and social distancing restrictions on laboratory utilization, community vision screening events, and international ophthalmology trips have significantly limited medical student accessibility to ophthalmology. Thus, medical students who had not identified mentors, completed research or gained additional exposure through community service arguably faced, and may continue to face, greater COVID-19-related impacts.

RV made several adjustments. There is typically a deadline to apply to participate in the program; in 2020, all interested URiM students were accepted into the program throughout the course of the summer and invited to participate in all RV events. At the end of the 2020 summer, we had 80 participants. The presentations were limited to 10 oral presenta- tions and 10 poster presentations. These presentations were judged at a live virtual event a week prior to the NMA scientific assembly. The top oral and poster presentations in the medical student and trainee categories were subsequently presented at the virtual NMA Ophthalmology Section scientific meeting. The traditional RV professional development and mentoring components were completed virtually as well.

In 2020, 29 RV medical student participants applied for ophthalmology residency and 5 resident participants applied for fellowships in retina, glaucoma, and oculoplastics. Twenty-two of the 29 applicants identified as Black/African American. For comparison, the average total number of Black/African Americans who registered for SF Match between 2016 and 2020 was 23.8 per year. Three RV applicants identified as Latinx, compared with 54.2 per year on average, registered for SF Match between 2016 and 2020. There is no corollary of the NMA Ophthalmology Section for interested Latinx; therefore, the RV program expanded its mission to increase recruitment of all URiM students. The average USMLE Step 1 score of the RV applicants in 2020 was $232.5 \pm 14.5$. This was greater than the national averages for USMLE Step 1 score among Black/African American (217) and Latinx (221) examinees. ${ }^{16}$ The average USMLE Step 1 score for ophthalmology applicants from 2016 to 2020 who did and did not match was 244.2 and 229.6 , respectively. In addition to their metrics, the RV students who applied for ophthalmology residency in 2020 were AOA and Gold Humanism honorees, as well as accomplished clinical and basic science researchers, inventors, musicians, and athletes. Twenty-four of 29 applicants matched; this includes 19 of 22 Black/African American and 3 of 3 Latinx applicants.

\section{Rabb-Venable Program Expansion}

The migration to a virtual format provided the opportunity to significantly expand the RV mentorship component. Additional offerings included: (1) panel discussions with RV junior faculty and trainee alums, (2) near-peer mentoring to assist with RV presentations and SF Match application preparation, (3) residency application review and feedback from URiM faculty volunteers, (4) mock residency interviews with Tours for Diversity, and (5) the Rabb-Venable Fireside Chat Series. The Fireside Chat Series provided virtual introductions to and informational sessions with individual ophthalmology residency program officials.

To develop the Fireside Chat Series, an invitation was sent via the Association of University Professors of Ophthalmology (AUPO) program directors listserv requesting that program directors, medical student educators, or other suitable program officers spend 30 to 45 minutes interacting with RV students, highlighting their programs' strengths and desired traits in qualified applicants. Twenty-nine programs participated. The "chats" were held between August 4, 2020, and August 31, 2020. There were two consecutive 1-hour sessions held on Monday through Thursday nights. Five of the 29 programs participated in a shorter format, 30-minute sessions in rapid succession on Saturday, August 15, 2020. 
Program representatives generally included a program or associate program director. However, some programs invited department chairs, medical student educators, vice chairs of education, diversity officers, and current trainees.

On average, 24 of the $29 \mathrm{RV}$ participants applying to SF Match in 2020 participated in each session. Participation was limited primarily by the students' academic and clinical responsibilities. The chats provided opportunities for program officers to get to know the URiM students applying to ophthalmology. Finally, the unfolding civil unrest surrounding the deaths of Ahmad Arbury, George Floyd, Daniel Prude, and the shooting of Jacob Blake led to impromptu discussions after meeting with the programs on the health care disparities exacerbated by the COVID-19 pandemic, the history of race in America, and racial health care disparities specific to ophthalmology. Twenty of the 29 programs that participated in the Fireside Chats interviewed three or more RV participants (unpublished internal survey). Thirteen programs matched 17 applicants who participated in the Fireside Chats.

\section{A Look Ahead}

The 2020 RV cohort was the largest and arguably the most competitive class of participants RV has hosted. They are a talented group who has performed well on USMLE Step 1. They are compassionate physicians dedicated to addressing health disparities in ophthalmology by providing excellent clinical care and engaging in population health research. RV has served as a natural community to support their professional development as ophthalmologists. These candidates will expand the program's ability to recruit increasing numbers of URiM physicians to the ophthalmology workforce. Their presence within the workforce will expand access to care and the capacity of the field to address racial disparities in eye health outcomes through research and exceptional clinical care.

RV stands committed to providing URiM ophthalmology candidates and trainees with ever-expanding resources to support the development and advancement of their careers. The migration to virtual meetings has provided an opportunity for RV to coordinate mentorship activities with program participants year-round, thus expanding the availability of mentorship opportunities for URiM students who lack sufficient exposure to ophthalmology in their communities and at their local medical schools.

Despite the hurdles the pandemic has imposed, the reaffirmation by AUPO and SF Match stating "the reliance on USMLE scores disadvantages underrepresented in medicine applicants" and their commitment to "use screening and ranking methods that ensure the holistic and equitable review of all applicants" 24 are truly positive developments.

We recognize that URiMs need to enter medical school in greater numbers to more fully diversify the complete physician workforce; however, the disparity in URiM SF Match outcomes cannot be overlooked. If Black/African American and Latinx SF Match outcomes were equivalent to white outcomes, the number of Black/African American and Latinx physicians entering the ophthalmology workforce would increase by 70.8 and $25.5 \%$, respectively. The RV and MOM programs continue to expand their efforts to recruit URiM students and prepare them to be successful candidates for ophthalmology residency; however, more work needs to be done to identify and mitigate the racial bias in the ophthalmology residency selection process.

\section{Funding}

National Eye Institute.

Conflict of Interest

None declared.

\section{References}

1 U.S. Department of Health and Human Services, Health Resources and Services Administration, National Center for Health Workforce Analysis. 2016. National and Regional Projections of Supply and Demand for Surgical Specialty Practioners: 2013-2025. Rockville, Maryland

2 Xierali IM, Nivet MA, Wilson MR. Current and future status of diversity in ophthalmologist workforce. JAMA Ophthalmol 2016; 134(09):1016-1023

3 Lam BL, Lee DJ, Zheng DD, Davila EP, Christ SL, Arheart KL. Disparity in prevalence of self-reported visual impairment in older adults among U.S. race-ethnic subgroups. Ophthalmic Epidemiol 2009;16(03):144-150

4 Elam AR, Lee PP. High-risk populations for vision loss and eye care underutilization: a review of the literature and ideas on moving forward. Surv Ophthalmol 2013;58(04):348-358

5 National Academies of Sciences, Engineering and Medicine. Making Eye Health a Population Health Imperative: Vision for Tomorrow. Washington, DC: The National Academies Press; 2016

6 Saydah SH, Gerzoff RB, Saaddine JB, Zhang X, Cotch MF. Eye care among US adults at high risk for vision loss in the United States in 2002 and 2017. JAMA Ophthalmol 2020;138(05):479-489

7 Anderson LM, Scrimshaw SC, Fullilove MT, Fielding JE, Normand JTask Force on Community Preventive Services. Culturally competent healthcare systems. A systematic review. Am J Prev Med 2003;24(3, Suppl):68-79

8 Snyder CR, Truitt AR. Exploring the provider preferences of multiracial patients. J Patient Exp 2020;7(04):479-483

9 Walker KO, Moreno G, Grumbach K. The association among specialty, race, ethnicity, and practice location among California physicians in diverse specialties. J Natl Med Assoc 2012;104; (1-2):46-52

10 Saha S, Komaromy M, Koepsell TD, Bindman AB. Patient-physician racial concordance and the perceived quality and use of health care. Arch Intern Med 1999;159(09):997-1004

11 Association of University Professors of Ophthalmology. (2020). Ophthalmology Residency Match Summary Report 2020. SF Match. Available at: https://www.sfmatch.org/SpecialtyInsideAll.aspx? $\mathrm{id}=6 \&$ typ $=2$ \&name $=$ Ophthalmology\#

12 Association of University Professors of Ophthalmology. (2020). Gender \& Ethnicity Data Ophthalmology Residency January 2020. Available at: https:/aupo.org/system/files/resources/2019-03/ 2019\%20AUPO\%20Residency\%20Gender-Ethnicity-Summary\% 20Final.pdf

13 Association of American Medical Colleges. (2019). AAMC Diversity in Medicine: Facts \& Figures 2016. Available at: https://www. aamcdiversityfactsandfigures2016.org/

14 Moxon NR, Goyal A, Giaconi JA, et al. The state of ophthalmology medical student education in the United States: an update. Ophthalmology 2020;127(11):1451-1453 
15 Linz MO, Jun AS, Clever SL, Lawson SM, Sanyal A, Scott AW. Evaluation of medical students' perception of an ophthalmology career. Ophthalmology 2018;125(03):461-462

16 Rubright JD, Jodoin M, Barone MA. Examining demographics, prior academic performance, and United States Medical Licensing Examination score. Acad Med 2019;94(03):364-370

17 Fernandez C, Lopez BL, Kushner M, Leiby BE, Den RB. Overemphasis of step 1 scores may affect applications pool diversity in radiation oncology. Pract Radiat Oncol 2020;10(01): e3-e7

18 Jarman BT, Kallies KJ, Joshi ART, et al. Underrepresented minorities are underrepresented among general surgery applicants selected to interview. J Surg Educ 2019;76(06): e15-e23

19 Poon S, Nellans K, Rothman A, et al. Underrepresented minority applicants are competitive for orthopaedic surgery residency programs, but enter residency at lower rates. J Am Acad Orthop Surg 2019;27(21):e957-e968
20 Edmond MB, Deschenes JL, Eckler M, Wenzel RP. Racial bias in using USMLE step 1 scores to grant internal medicine residency interviews. Acad Med 2001;76(12):1253-1256

21 Spector AR, Railey KM. Reducing reliance on test scores reduces racial bias in neurology residency recruitment. J Natl Med Assoc 2019;111(05):471-474

22 Williams M, Kim EJ, Pappas K, et al. The impact of United States Medical Licensing Exam (USMLE) step 1 cutoff scores on recruitment of underrepresented minorities in medicine: a retrospective cross-sectional study. Health Sci Rep 2020;3(02):e2161

23 Coalition for Physician Accountability. Final report and recommendations for medical education institutions of LCMEaccredited, U.S. osteopathic, and non-U.S. medical school applicants. Available at: https://www.aamc.org/system/files/2020-05/ covid19_Final_Recommendations_05112020.pdf

24 Quillen DA, Siatkowski RM, Feldon Sof the Association of University Professors of Ophthalmology. COVID-19 and the ophthalmology match. Ophthalmology 2021;128(02):181-184 